\title{
CONTRIBUCIÓN AL ESTUDIO FITOQUÍMICO DE LA ESPECIE “Sansevieria trifasciata Prain” Y SU COMPORTAMIENTO FRENTE A ENSAYOS BIOLOGICOS
}

\section{CONTRIBUTION TO PHYTOCHEMICAL STUDY OF THE SPECIES “Sansevieria trifasciata prain" AND THEIR BEHAVIOR IN BIOLOGICAL ASSAYS}

\author{
Ludy Molina-Fajardo ${ }^{1-2}$, Milton Gómez-Barreraa, Jhon Carlos Castaño-Osorio ${ }^{2^{*}}$ \\ a - Laboratorio Búsqueda de Principios Bioactivos \\ b- Grupo de Inmunología Molecular GYMOL. Facultad de ciencias de la Salud. Universidad del Quindío. \\ Recibido: Octubre 25 de 2010 \\ Aceptado: Diciembre 12 de 2011
}

*Autor de Correspondencia: Carrera 15 calle 12 Norte. Armenia. Correo electrónico: gymol@uniquindio.edu.co

\section{RESUMEN}

Se evaluaron las diferentes propiedades biológicas de los extracto total, etanólico y la fracción alcaloidal de hojas de Sansevieria trifasciata, como la actividad antimicrobiana, contra Staphylococcus aureus, Escherichia coli y Candida albicans y la actividad citotóxica en células Vero y antitumoral en mieloma murino y Hep G2. Asimismo, se les realizo tamizaje fitoquímico para determinar los metabolitos secundarios presentes en las hojas y se determinó la toxicidad en Artemia salina. Se pudo determinar la presencia de alcaloides, leucoantocianidinas, saponinas esteroidales, cumarinas, lactonas sesquiterpénicas, glicósidos cardiotónicos, carotenoides y carbohidratos. No se detectó presencia de flavonoides, nafto y/o antroquinonas y taninos. No se encontró actividad antibacteriana contra E.coli, sin embargo el extracto etanolico mostro actividad frente a S.aureus y C. Albicans. Ninguno de los extractos mostraron actividad citotoxica frente a células Vero ni anti tumoral en células de mieloma de ratón. La fracción alcaloidal presento actividad anti tumoral en células Hep-G2. La concentración letal media en Artemia salina de los extractos fue de $146,1 \mu \mathrm{g} / \mathrm{mL}$.

Palabras Claves: Sansevieria trifasciata Prain, Staphylococcus aureus, Escherichia coli, Candida albicans, Celulas Vero, Células Hep G2, Mieloma Murino.

\begin{abstract}
The present study evaluated the different biological properties of the total ethanolic extract and the alkaloid fraction obtained from leaves of Sansevieria trifasciata, such as the antimicrobial activity against Staphylococcus aureus, Escherichia coli and Candida albicans, and cytotoxic activity on Vero cells; and antitumor activity in murine myeloma and HepG2. Likewise, phytochemical screening was conducted to determine some secondary metabolites present in the leaves and the toxicity was measured with Artemia salina. It was possible to determine the presence of alkaloids, leucoanthocyanidins, steroidal saponins, coumarins, sesquiterpene lactones, glycosides cardiac, carotenoids and carbohydrates. There was no presence of flavonoids, naphtha and/or anthroquinones and tannins. No antibacterial activity was found against $E$. coli, but the ethanol extract showed activity against $S$. aureus and $C$. Albicans. None of the extracts showed cytotoxic activity against Vero cells or antitumor mouse myeloma cells. The alkaloid fraction contained antitumor activity in HepG2 cells. The mean lethal concentration in Artemia salina extracts was $146.41 \mathrm{mg} / \mathrm{mL}$.
\end{abstract}

Key words: Sansevieria trifasciata Prain, Staphylococcus aureus, Escherichia coli, Candida albicans, Cells Vero, cells HepG2, mouse myeloma.

\section{INTRODUCCIÓN}

Las plantas han sido desde la antigüedad un recurso al alcance del ser humano para su alimentación y la curación de sus enfermedades; en la actualidad cientos de plantas son utilizadas en la medicina, pero la ciencia moderna analizando y estudiando los efectos terapéuticos de las plantas, quiere precisar, comparar y clasificar las diversas propiedades, con el fin de conocer los principios activos responsables de eliminar o aliviar 
enfermedades, separarlos los compuestos de las plantas que lo contienen, determinar sus estructuras químicas, procurar su síntesis, proponer modificaciones estructurales en busca de una mayor actividad, finalmente dar a conocer a la humanidad los resultados de los estudios.

La utilización de las plantas en medicina no ha perdido interés, según lo demuestra el hecho que durante alrededor de quince años (1959-1973), en el mercado de los Estados Unidos el 25,0\%(1), de las prescripciones médicas contenían principios derivados de plantas (no menor de $24,0 \%$, ni mayor de $26,0 \%$ ); esta estabilidad permite presumir que el porcentaje no es diferente en estos momentos (reportes de esta naturaleza no ha sido posible encontrar a nivel nacional). Es también frecuente encontrar en estas prescripciones médicas, extractos crudos de plantas, aunque la proporción es menor, alrededor de 2,5\%(1).

La especie Sansevieria trifasciata Prain ha despertado interés ya que desde la antigüedad se ha utilizado en la industria por su importancia económica en la producción de jabones y detergentes, esto se debe a la cantidad de saponinas contenidas en esta especie. Además es conocida como una de las plantas de interior más resistentes que hay. Tiene fama de indestructible. Resiste la atmósfera seca y caliente de las habitaciones, la luz pobre, el olvido del riego, años sin trasplantar, las plagas y enfermedades, etc. (2).

Dado que aun no se conoce la actividad farmacológica Sansevieria trifasciata de Prain, nos propusimos como objetivo evaluar las diferentes propiedades biológicas de los extracto total etanólico y la fracción alcaloidal de hojas de Sansevieria trifasciata como son la actividad antimicrobina frente a E.coli, S.aureus, Candida Albicans , actividad anti neoplasica frente a mieloma murino Sp2/O-Ag14 y células Hep G2, citotoxicidad en células vero, concentración letal media (CL50) de estos frente a Artemia salina.

\section{MATERIALES Y MÉTODOS}

Tamizaje fitoquímico preliminar y preparación de los extractos

El tamizaje fitoquímico preliminar se fundamento en los parámetros establecidos por Sanabri-Galindo 1983(3). Los pasos fueron los siguientes:

1. Obtención del extracto etanólico total.
2. Análisis fitoquímico preliminar del extracto etanólico total de la planta.

3. Análisis preliminar de alcaloides.

4. Obtención de los extractos para el análisis de esteroides y/o metil-esteroles libres, naftoquinonas y/o antraquinonas, taninos y saponinas.

5. Obtención de los extractos para el análisis de cumarinas, lactonas sesquiterpenicas y glicósidos cardiotónicos.

\section{Determinación de actividad antimicrobiana}

Para las pruebas de actividad biológica se utilizaron el extracto total etanolico y la fracción alcaloidal de hojas de Sansevieria trifasciata. Los extractos fueron desecados mediante evaporación en baño de María, obteniéndose una pasta de cada uno de los extractos. Se preparo una solución estándar de cada uno de los extractos después de desecados a uno concentración de $2000 \mu \mathrm{g} / \mathrm{ml}$ (p/v), preparado en una solución de DMSO y agua destilada. Posteriormente se realizaron diluciones dobles seriales de cada uno de los extractos $(1000 \mu \mathrm{g} / \mathrm{ml}$. ,500 $\mu \mathrm{g} / \mathrm{ml} ., 250 \mu \mathrm{g} / \mathrm{ml} ., 125 \mu \mathrm{g} / \mathrm{ml}, 62,5$ $\mu \mathrm{g} / \mathrm{ml}, 31,25 \mu \mathrm{g} / \mathrm{ml}$., $15,625 \mu \mathrm{g} / \mathrm{ml}, 7,8125 \mu \mathrm{g} / \mathrm{ml})$, las cuales se utilizaron en los diferentes ensayos de actividad biológica in vitro. Se utilizaron para la evaluación de la actividad antimicrobiana de extracto total etanólico y la fracción alcaloidal de hojas de Sansevieria trifasciata, los siguientes microorganismos:

- Staphylococcus aureus (S. a) aislado clínico

- Escherichia coli (E. c) ref. BL 21

- Candida albicans (C. a) ref. ATCC 90028

El Staphylococcus aureus se obtuvo de aislados clínicos identificados en el Centro de Investigaciones Biomédicas de la Universidad del Quindío. Se recultivaron en agar sangre, incubadas a $37^{\circ} \mathrm{C}$ durante 24 horas. En relación a Candida albicans fue cultivada en agar Sabouraud a $37^{\circ} \mathrm{C}$ durante 48 horas.

Para la determinación de la actividad antimicrobiana se realizo por el método de microtitulación en placa con indicador de resazurin (Resazurin sal sódica).

\section{Preparación del inóculo}

Se preparó y esterilizó el medio de cultivo, se incubo a una temperatura de 37 으 por 12 h, se tomó el asa estéril e inoculó un par de colonias por especie en tubos de ensayo que contenían $3 \mathrm{ml}$. De caldo de tripticasa de soya preparado con anterioridad para evitar contaminación, se incubo a 37으 por $2 \mathrm{~h}$. 
La suspensión utilizada para Staphylococcus aureus, Candida albicans y Escherichia coli fue $5 \times 10^{5} \mathrm{ufc} / \mathrm{ml}$, Se estandarizo a una D.O. de 0,4 a $620 \mathrm{~nm}$. se realizó el cultivo de la suspensión bacteriana o de hongos en 100 $\mu \mathrm{L}$ de los medios antes descritos en placas de microtitulación NUNC estériles de 96 pozos con $50 \mu \mathrm{L}$ de la solución acuosa-DMSO de cada uno de los extractos y $5 \mu \mathrm{L}$ de resazurin, como controles de la actividad antimicrobiana se utilizo una solución de antibiótico/antimicótico (10.000 unidades $/ \mathrm{mL}$ de Gentamicina y $25 \mu \mathrm{g} / \mathrm{mL}$ de Anfotericina B) $(4,5,6)$.Las placas fueron incubadas a $37{ }^{\circ} \mathrm{C}$ durante 24 horas.

El resazurin es un indicador de oxido-reducción el cual es de color azul en medio estéril, y cambia a color rosa cuando es reducido por la oxido-reductasa producidas por las células viables (4).

Debido a que el método es una titulación, donde el indicador es resazurin, el analito los microorganismos y el valorante el extracto o la fracción, se leyó la densidad óptica en un lector de microelisa (DYNATECH MR5000) a las 13 y $23 \mathrm{~h}$ a una longitud de onda de $630 \mathrm{~nm}$.

\section{DETERMINACIÓN DE ACTIVIDAD CITOTÓXICA Y ANTITUMORAL}

Las células utilizadas fueron células Vero que son aisladas del epitelio de riñón de mono verde africano adulto, (ATCC-CCL81), las células Hep G2(ATCC No. HB8065 ) se cultivaron en medio DMEM (Invitrogen ${ }^{\circledR}$ ), suplementado con $10 \%$ de suero fetal bovino (Invitrogen ${ }^{\circledR}$ ) inactivado con calor, antibióticos(Penicilina G 10000 unidades/ml, Sulfato de estreptomicina $10 \mathrm{mg} / \mathrm{ml}$ )-antimicótico (Anfotericina B $25 \mu \mathrm{g} / \mathrm{ml}$ ) y L-Glutamina $200 \mathrm{mM}$. Las células de mieloma de ratón Sp2/O-Ag14 (ATCC:CRL1581) $y$, se cultivaron en medio RPMI 1640 (Invitrogen ${ }^{\circledR}$ ) suplementado de igual forma que el medio de cultivo de las células Vero.

Se utilizaron como métodos para la evaluación de la citotoxicidad en células Vero y actividad antitumoral en células de mieloma murino Sp2/O-Ag14 la adición del colorante Azul de alamar en cada uno de los pozos de cultivo. Para lo cual en cada pozo de cultivo de las placas de 96 pozos se cultivaron 10000 células. Cada ensayo se realizó por triplicado, haciendo lectura a las 6 y $23 \mathrm{~h}$. con lector de microelisa(DYNATECH MR5000) a una longitud de onda de $630 \mathrm{~nm}$. También se interpretaron los resultados de las placas mediante la observación de cambio de coloración de azul a rosa que indica la actividad enzimática de las células en división (7).

En ambos métodos se utilizo la técnica de microtitulación en pozo del extracto etanólico a diferentes concentraciones $(1000 \mu \mathrm{g} / \mathrm{ml}$, $500 \mu \mathrm{g} / \mathrm{ml}$., $250 \mu \mathrm{g} / \mathrm{ml} ., 125 \mu \mathrm{g} / \mathrm{ml}, 62,5 \mu \mathrm{g} / \mathrm{ml}$ ).

\section{Determinación de la concentración letal media (CL50)}

La determinación preliminar de la dosis letal media en Artemia salina se realizó por exposición de 10 crustáceos a concentraciones de $1000 \mu \mathrm{g} / \mathrm{ml}, 500$ $\mu \mathrm{g} / \mathrm{ml}, 250 \mu \mathrm{g} / \mathrm{ml}, 125 \mu \mathrm{g} / \mathrm{ml}, 62,5 \mu \mathrm{g} / \mathrm{ml}, 31,25 \mu \mathrm{g} / \mathrm{ml}$, $15,625 \mu \mathrm{g} / \mathrm{ml}, 7,8125 \mu \mathrm{g} / \mathrm{ml}$ de los extractos, teniendo en cuenta el conteo de larvas muertas luego de 24 horas de inicio del procedimiento, que se realizó por triplicado. Además se realizó el conteo de larvas muertas en el blanco para descartar una posible interferencia del solvente $\left(\mathrm{H}_{2} \mathrm{O}\right.$ y DMSO $\left.2 \%\right)$ en la prueba, debido a que en la preparación de los extractos se uso esta proporción de DMSO. De esta manera podemos descartar la incidencia del DMSO en el ensayo.

\section{Eclosión de crustáceos}

Los huevos de Artemia franciscana se hicieron eclosionar en agua de mar(solución de sal marina al $2.5 \%, \mathrm{p} / \mathrm{v}$ ) durante 24 horas. Luego los nauplios obtenidos fueron expuestos a distintas concentraciones de los diferentes extractos de la planta $(8,9,10)$.

Los nauplios muertos fueron contados a las 24 horas de exposición a los diferentes extractos y fueron calculados los porcentajes de mortalidad, la CL50 se evalúo mediante el análisis probit(10-12), con intervalos de confianza al 95\%. Por cada extracto se hicieron cuatro ensayos, cada uno por triplicado.

\section{Pruebas estadísticas}

La técnica de análisis estadístico utilizada fue ANOVA de dos factores con interacciones, para analizar el efecto de la concentración y el tiempo en la densidad óptica, en forma separada para cada extracto en cada uno de los ensayos para microorganismos y las líneas celulares. Los datos obtenidos se calcularon en el programa STATGRAPHIS CENTURION XV.

\section{RESULTADOS Y DISCUSIÓN}


Tamizaje fitoquímico preliminar: En el tamizaje fitoquímico se obtuvo la presencia de alcaloides, saponinas, esteroides, triterpenos y lactonas sesquiterpénicas(Ver Tabla 1), las cuales al relacionarlos y compararlos nos concuerda con los estudios previos de la familia y de la especie(13).

Tanto los resultados positivos como los negativos se corroboraron a través de cromatografía de capa delgada, según el tipo de metabolito buscado. Al hacer la comparación con otras especies de la misma familia se pueden observar la similitud de metabolitos secundarios encontrados en las hojas de $S$. trifasciata, los cuales han sido de gran utilidad en el momento de una clasificación taxonómica, por lo que ha sido uno de los mayores problemas de esta planta, al tener características morfológicas muy similares a la familia Agavaceae como ha sido reportada en algunas investigaciones.

Al hacer el seguimiento cromatografíco en capa delgada de las fracciones obtenidas de la cromatografía en columna de la fracción diclorometanólica se pudo demostrar la presencia de lactonas sesquiterpénicas

Tabla . Resultado general del tamizaje fitoquímico propuesto por Sanabria para extracto etanólico de hojas de S. Trifasciata Prain.

\begin{tabular}{|c|c|c|c|c|c|}
\hline \multirow{2}{*}{$\begin{array}{l}\text { Sustancia } \\
\text { Alcaloides }\end{array}$} & \multirow[t]{2}{*}{ Prueba } & \multicolumn{4}{|c|}{ Resultado } \\
\hline & & $\begin{array}{l}\text { Solubles } \\
\text { en } \mathrm{CHCl}_{3}\end{array}$ & $\begin{array}{c}\text { Solubles } \\
\text { en } \mathrm{CHCl}_{3}- \\
\mathrm{ETOH}\end{array}$ & $\begin{array}{l}\text { Alcaloides } \\
\text { fenólicos }\end{array}$ & $\begin{array}{c}\text { Amonio } 4^{0} \\
\text { u óxidos } \\
\text { de aminas }\end{array}$ \\
\hline & Dragendorff & ++ & +++ & ++ & + \\
\hline & Mayer & +++ & + & + & ++ \\
\hline & Valser & + & +++ & + & ++ \\
\hline & Scheibler & +++ & +++ & + & + \\
\hline \multirow[t]{2}{*}{ Flavonoides } & Cianidina & \multicolumn{4}{|c|}{-} \\
\hline & $\mathrm{HCl}$ & \multicolumn{4}{|c|}{++} \\
\hline Nafto y/o & Borntragger & \multicolumn{4}{|c|}{-} \\
\hline \multirow[t]{2}{*}{ Antraquinonas } & $\mathrm{H}^{+}$ & \multicolumn{4}{|c|}{-} \\
\hline & $\mathrm{OH}^{-}$ & \multicolumn{4}{|c|}{-} \\
\hline \multirow[t]{3}{*}{ Taninos } & Gelatina sal & \multicolumn{4}{|c|}{+++} \\
\hline & $\mathrm{FeCl}_{3}$ & \multicolumn{4}{|c|}{-} \\
\hline & $\begin{array}{l}\text { Acetato de } \\
\text { plomo }\end{array}$ & \multicolumn{4}{|c|}{+++} \\
\hline \multirow[t]{3}{*}{ Saponinas } & Hemólisis & \multicolumn{4}{|c|}{++} \\
\hline & Espuma & \multicolumn{4}{|c|}{++} \\
\hline & Vainillina & \multicolumn{4}{|c|}{+++} \\
\hline \multirow{2}{*}{$\begin{array}{c}\text { Esteroides y/o } \\
\text { Triterpenos }\end{array}$} & $C C D^{2}$ & \multicolumn{4}{|c|}{+++} \\
\hline & $\begin{array}{l}\text { Lieberman- } \\
\text { Burchard }\end{array}$ & \\
\hline \multirow[t]{2}{*}{ Cumarinas } & Placa CCD & \multicolumn{4}{|c|}{+} \\
\hline & $\begin{array}{c}\text { Hidroxamato } \\
\mathrm{Fe}^{3+}\end{array}$ & & & & \\
\hline \multirow[t]{4}{*}{ Cardiotónicos } & Placa CCD & \multicolumn{4}{|c|}{++} \\
\hline & vainillina & & & & \\
\hline & Placa CCD & \multicolumn{4}{|c|}{++} \\
\hline & Baljet & & & & \\
\hline Lactonas & Placa CCD & \multicolumn{4}{|c|}{+++} \\
\hline sesquiterpénicas & Vainillina & & & & \\
\hline \multirow[t]{3}{*}{ Carbohidratos } & Tollens & \multicolumn{4}{|c|}{+++} \\
\hline & Molish & \multicolumn{4}{|c|}{+++} \\
\hline & Fehling & \multicolumn{4}{|c|}{+++} \\
\hline
\end{tabular}




\section{Determinación de actividad antimicrobiana}

Actividad contra E. coli, como se puede observar el figura 1 , se nota que no hubo actividad en los efectos de la fracción alcaloidal y al compararlo con el extracto etanólico frente al antibiótico, por lo que se puede decir que ninguno de estos dos tiene efecto de inhibición de crecimiento en la cepa de $E$. coli.

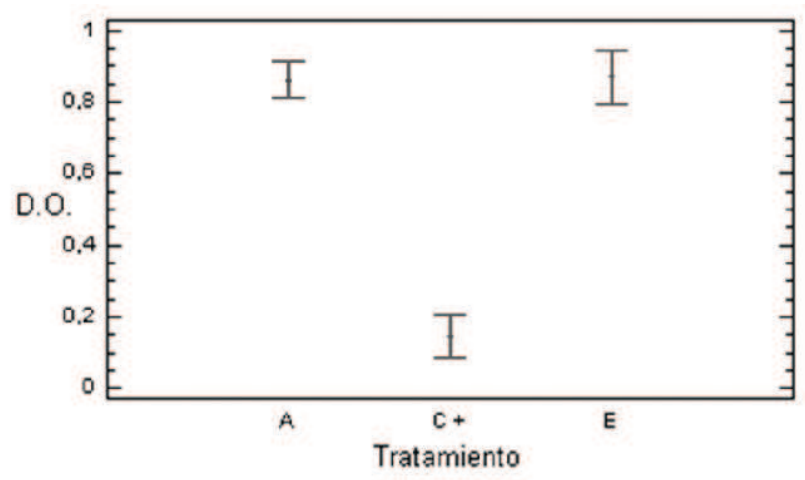

Figura1 . Comparación de la actividad del extracto etanólico y la fracción alcaloidal respecto al antibiótico frente a E. coli. A.fracción alcaloidal, $C+$ tratamiento con antibiótico de actividad conocida, $E$. extracto etanolico. A. Extracto alcaloidal, C+. Control positivo con tratamiento con antibióticos. E. Extracto Etanolico.

Actividad contra .S.aureus, En el figura 2 podemos observar que las concentraciones que dieron mayor inhibición, tanto de la fracción como del extracto, tienen un comportamiento algo similar al del antibiótico, lo cual se puede decir que tiene un ligero efecto inhibitorio en la cepa de $S$. aureus.

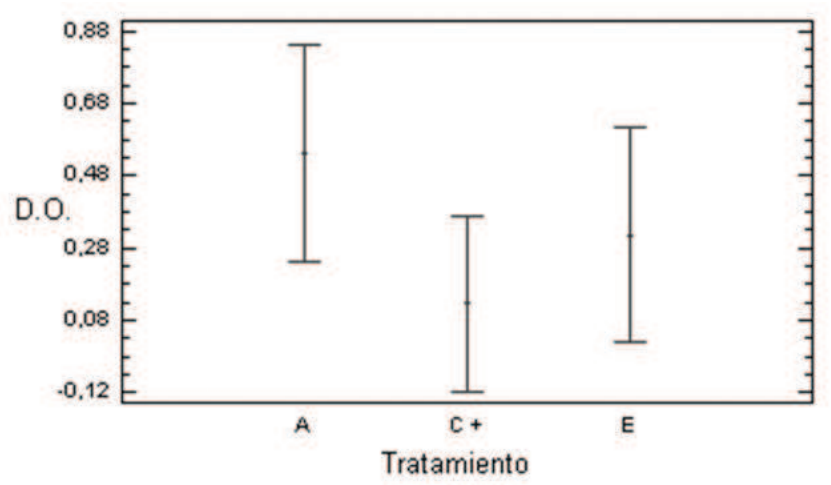

Figura 2. Comparación de la actividad del extracto etanólico, la fracción alcaloidal respecto al antibiótico frente a S.aureus. A. Extracto alcaloidal, C+. Control positivo con tratamiento con antibióticos. E. Extracto Etanólico.

\section{Actividad contra C.albicans,}

En el caso de $C$. albicans solo se encontró efecto inhibitorio en el extracto etanólico, este caso el agente inhibitorio no son los alcaloides, si no otro tipo de metabólito, como las de saponinas, las cuales saponifican la superficie celular hidrofobica de las levaduras (Kazanjian y Fariñas, 2006) y las deja a merced de un choque osmótico.

Figura 3. Comparación de la actividad del extracto etanólico y la

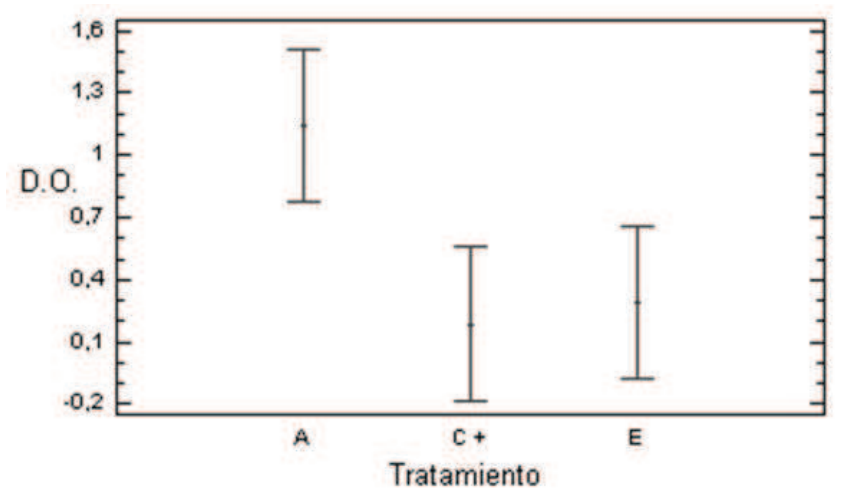

fracción alcaloidal respecto al antibiótico frente a E. coli. A.fracción alcaloidal, C+ tratamiento con antibiótico de actividad conocida, E. extracto etanolico..

Por otra parte, se encontró que ni el extracto, ni la fracción alcaloidal inhibió el crecimiento de $E$. coli, es conocido que bacterias Gram negativas como estas, poseen una membrana adicional denominada "Estructura OM" lo que confiere un mayor grado de resistencia a los agentes antimicrobianos(15).

Por ello se puede explicar la inhibición de crecimiento en $S$. aureus, para la fracción y el extracto. La identificación de terpenos en el vegetal, revelan la conexión entre la actividad determinada y su composición química, estos atraviesan la barrera superficial de la bacteria y después se fijan sobre su membrana celular, que afirma la inhibición de crecimiento del S. aureus(14). Bioensayos realizados en otras plantas como en el Aloe vera reportado por Martínez que utilizaron cuatro bacterias (Grampositivas y Gram negativas) y una levadura, encontrando solo una ligera actividad inhibitoria en $S$. aureus(16), Ávila y otros relacionan la acción antibacteriana frente $S$. aureus a la presencia de terpenos y flavonoides en la especie Diplostephium tolimense, al aumentar la simplicidad química del 
extracto aumenta la inhibición (15), esto también podría relacionarse al resultado obtenido, ya que en la especie S. trifasciata también se encontró gran presencia de terpenos. En la evaluación preliminar de Sida rhombifolia L. realizada por Bruges y Reguero se puede observar resultados similares a los obtenidos, por lo que en $E$. coli no encontraron ningún efecto, al contrario de $S$. aureus, este resultado se le atribuyo a los metabolitos encontrados que fueron flavonoides $y$ terpenos(17).

Determinación de actividad citotoxica y antitumoral En la tabla 2 se puede observar que en el extracto etanólico ni en la fracción alcaloidal existen diferencias signifacativas en los valores de absorbancia obtenidos, infiriendo que no hay ningún tipo de efecto tanto en las concentraciones como en el tiempo, sin embargo la fracción alcaloidal muestra un mayor índice de letalidad en las células de Hep G2 al compararlas con las Vero, aunque este valor no es muy significativo.

Las pruebas realizadas en la determinación de actividad citotóxica en células vero y antineoplásica en mieloma murino y Hep G2, solo mostraron inhibición en las células Hep G2 en la

Tabla 2. D.O. a las 6 y $23 \mathrm{~h}$ en el extracto y la fracción en células Hep G2

\begin{tabular}{lllll}
\hline concentración $(\mu \mathrm{g} / \mathrm{mL})$ & Alcal oidal $\mathbf{6} \mathbf{h}$ & Alcaloidal $\mathbf{2 3} \mathbf{~}$ & Etanólico $\mathbf{~ h}$ & Etanólico $\mathbf{2 3} \mathbf{~}$ \\
\hline $\mathbf{1 0 0 0}$ & 0,36133 & 0,40433 & 0,30266 & 0,30366 \\
$\mathbf{5 0 0}$ & 0,288 & 0,277 & 0,22033 & 0,312 \\
$\mathbf{4 0 0}$ & 0,29833 & 0,24 & 0,201 & 0,451 \\
$\mathbf{3 0 0}$ & 0,259 & 0,236 & 0,199 & 0,30466 \\
$\mathbf{2 0 0}$ & 0,23066 & 0,18666 & 0,30066 & 0,48166 \\
$\mathbf{1 0 0}$ & 0,22933 & 0,19033 & 0,376 & 0,24366 \\
\hline
\end{tabular}

fracción alcaloidal, debido a que estos poseen una marcada capacidad para concentrarse selectivamente en el núcleo celular,intercalarse dentro del ADN y bloquear al ciclo celular al inicio de la fase de replicación, otro modo de acción consiste en inhibir la topoisomerasa I enlazándose al complejo covalente Topo-I-ADN con la formación de un ducto que causa la estabilización de esté e impide el re-enrollamiento de la hebra de $\operatorname{ADN}(18)$

\section{Determinación de la concentración letal media (CL50)}

La dosis letal media fue de $146,141 \mu \mathrm{g} / \mathrm{mL}$ en las hojas de $S$. trifasciata en A.salina, al compararlo con los resultados en $S$. ehrenbergii en frutos obtenido por Al-Fatimi y colaboradores fue de $50 \mu \mathrm{g} / \mathrm{mL}$ que indica una mayor toxicidad, por lo que se necesita una menor concentración para eliminar la mitad de la población(19).

\section{Conclusiones}

- Las hojas de la especie Sansevieria trifasciata Prain, presenta alcaloides, leucoantocianidinas, saponinas esteroidales, cumarinas, lactonas sesquiterpénicas, glicósidos cardiotónicos, carotenoides y carbohidratos.

- En esta especie no se detectó presencia de flavonoides, nafto y/o antroquinonas y taninos

- El extracto etanólico y las fracción alcaloidal no presentaron actividad antibacteriana contra $E$. coli

-El extracto etanólico tiene mayor efecto de inhibición que la fracción alcaloidal frente a $S$. aureus.

- El extracto etanólico tiene propiedades antimicóticas frente a C.albicans.

- La fracción alcaloidal no ejerce ningún efecto de inhibición en la cepa de $C$. albicans.

- No se presento actividad antitumoral en las células de mieloma murino. 
- El extracto etanolico ni la fracción alcaloidal mostraron acción citotóxicidad frente a las células Vero.

- En la fracción alcaloidal se encontró una mayor actividad antitumoral que en el extracto total en la línea celular Hep G2.

- La concentración letal media es de 146.41 g/mL en las hojas de S.trifasciata.

\section{BIBLIOGRAFIA}

1.Gonzalez D. Plantas medicinales: un resumen de farmacognosia. 2 ed. Bogota (1980), editorial tercer mundo.

2. Mimaki Y, Inoue T, Kuroda M and Sashida Y. Steroidal Saponins from Sansevieria Trifasciata. Phytochemistry.1996; 43(6):1325-31.

3. Sanabria A. Análisis fitoquímico preliminar: Metodología y su aplicación en la evaluación de 40 plantas de la familia Compositae. Santa fe de Bogotá (1983); trabajo de grado. Universidad Nacional de Colombia. Facultad de Química Farmacéutica

4.Sarker SD, Nahar L, and Kumarasamy Y. Microtitre plate-based antibacterial assay incorporating resazurin as an indicator of cell growth, and its application in the in vitro antibacterial screening of phytochemicals Methods. 2007; 42(4): 321-24.

5. Rahman, M. M. and Gray, A. I. A benzoisofuranone derivative and carbazole Alkaloids from Murraya koenigii and their antimicrobial activity. Phytochemistry. 2005;66 (13): 1601-06.

6. Rahman M M, Gray A I, Khondkar P, Sarker S D. Antibacterial and Antifungal Activities of the Constituents of Flemingia paniculata. Pharmaceutical Biology.2008;46(5) 356-59.

7. Al-Nasiry S, Geusens N, Hanssens M, Luyten C and Pijnenborg R. The use of Alamar Blue assay for quantitative analysis of viability, migration and invasion of choriocarcinoma cells.. Human reproduction.2007;22(5): 1304-9.

8. Krishnaraju AV, Rao-Tayi VN, Sundararaju D, Vanisree M, Tsay HS, Subbaraju GV Assessment of bioactivity of Indian medicinal plant using brine shrimp (Artemia salina) Lethality Assay. Int. J.Appl. Sci. and Eng. 2005; 3(2): 125-34.

9. Vizcaino-Marquez RL y De la Rosoa C. Química toxicidad preliminar con Artemia salina y efecto del extracto acuoso de las hojas de Justicia secunda vahl (acantaceae) sobre la germinación en semillas de frijol (phaseolus vulgaris). Revista Dugandia, facultad de ciencias básicas. 2005;1(2):78-82.

10. Cantillo J, Güette J, Baldiris R, Jaramillo B, Olivero J. Evaluación de la toxicidad aguda (CL5O) frente a artemia franciscana y la actividad hemolitica de los extractos acuosos, en diclorometano y metanólico parcial de justicia secunda (VAHL.). Scientia Et Technica. 2007;13(33):257-58.

11. Finney, D. (1971). 3rd ed. Cambridge Univ. Press, Cambridge, UK. London and New York. pp.76-80.

12. Ogunnusi, T.A. and Dosumu, O. O. Bioactivity of crude extracts of Euphorbia kamerunica Pax using brine shrimp (Artemia salina) lethality assay. Journal of Medicinal Plants Research. 2008; 2(12):370-73. 
13. Garcia D y Medina M. Composición química, metabolitos secundarios, valor nutritivo y aceptabilidad relativa de diez árboles forrajeros.Zootecnia Trop. 2006; 24(3):233-50.

14. Tabares P. Avila L, Torres F, Cardona D, Quiñones W, Forero J, et al. metabolitos secundarios antivirales de algunas especies de la familia euphorbiaceae. Scientia et technica. 2007;13 (33):107-110.

15. Avila L, Baqueo E, Viña A, Murillo E.Actividad antibacteriana de diplostephium tolimense cuatrec. (asteraceae) frente a staphylococcus aureus. Vitae, revista de la facultad de química farmacéutica.2006;13(1):55-60.

16. Martínez MJ, Betancourt J, Alonso-González N, Jauregui A.Screening of some Cuban medicinal plants for antimicrobial activity. J Ethnopharmacol. 1996 5;52(3):171-4.

17. Bruges $\mathrm{K}$ y Reguero M T. Preliminary evaluation of Sida rhombifolia L. Revista Colombiana de biotecnología.2007;9(1):5-13.

18. Melendez C. y Kouznetsov V. Alcaloides: importancia biológica y esfuerzos sintéticos. Revista de la facultad de ciencias. Universidad Javeriana. 2005; 10(2): 5-18.

19. Al-Fatimi M, Martina W, Schröder $G$ and Lindequist U. Antioxidant, antimicrobial and cytotoxic activities of selected medicinal plants from Yemen. Journal of Ethnopharmacology.2007;111(3):657-66. 